\title{
OTRI3. COMPARATIVE MOLECULAR DOCKING ANALYSIS AMONG SIX SCFV MONOCLONAL ANTIBODIES AGAINST HEPATITIS B VIRUS SURFACE ANTIGEN.
}

Artur Hermano Sampaio Dias ${ }^{1}$; Márcia Arissawa ${ }^{2}$; Alexandre Bezerra Conde Figueiredo²; João Hermínio Martins da Silva ${ }^{1}$.

${ }^{1}$ Fiocruz-CE;

${ }^{2} \mathrm{BM}$.

INTRODUCTION Hepatitis B figures as one of the biggest public health problems worldwide. Thereafter, some effort should be dedicated in order to develop efficient diagnostic and therapeutic tools as based on the monoclonal antibodies technology. In this context, monoclonal antibody fragments, as scFv, present some advantages in immunotherapeutical applications. The scFv format consists of variable regions of heavy $(\mathrm{VH})$ and light $(\mathrm{VL})$ chains, which are joined together by a flexible peptide linker. The length of the flexible DNA linker and their amino acid composition plays an important role in the design of a viable linker peptide. The study of these aspects can lead to the improvement of the scFv activity.

OBJECTIVE This study aimed to evaluate the contribution of different linkers on the conformational structure of $\mathrm{scFv}$ fragments in order to evaluate the best in silico performance in a docking against HBsAg.

METHODOLOGY In this study, we carried out an in silico homology modeling of two distinct primary structures of $\mathrm{mAB}$ s: two scFv having the same Complementarity Determining Regions (CDRs) named clones A and B. The comparative modeling was carried out by the software Modeller 9.15, using Protein Data Bank tridimensional structures as templates. To each constructed clone, 3 different linkers were added separately: a small one, 8 residues long (GGGSGGGG) - HBCLA1 e HBCLB1 -, a large one, 15 residues long (GGGGSGGGGSGGGGS) - HBCLA2 e HBCLB2 and an intermediate, 10 residues long (GGGGSGGGGS) - HBCLA3 e HBCLB3. The POPS server was used to determine which CDR residues were active. The $\mathrm{HBs} A g$ active residues were identified by the 3DLigandSite server. All active residues were used as input in a molecular protein-protein docking in HADDOCK 2.2 server. Thus, HADDOCK score and Root Mean Square Deviation (RMSD) were compared 
amongst all six scFv-HBsAg clusters, aiming to find the lowest values for both parameters.

RESULTS The results showed that clone A, with a 10 residues-long linker (HBCLA3), was the best combination among all others: its score and RMSD were the lowest, respectively: $-134.2 \pm 0.7$ and $0.9 \pm 0.5$. Addtionally, the second best score was also from a clone A combination: HBCLA1, whose score was $-133.2 \pm 8.8$, but this combination (with a 8 residues-long linker) showed the worst RMSD: $24.4 \pm 0.0$. The lower scores depicts better interaction with the antigen.

CONCLUSION Based on the comparison among three different linkers in the $\mathrm{scFv}$ fragment by in silico analysis, it can be concluded that an intermediate-length linker is a good fit for the coupling between our scFv and $\mathrm{HBsAg}$, favouring the coverage of the binding pockets on HBsAg surface. Molecular dynamic analysis and alanine scanning are to be done in order to identify other structural features that can contribute to the improvement of the antibody-antigen interaction.

KEYWORDS scFv, Hepatitis B, linker peptide, molecular docking. 